\title{
Self-made 3D-printed encapsulation of thin-film transducers for reliable force measurement in biomedical applications
}

\author{
Pertusio Raffaele ${ }^{\mathrm{a}}$ and Roatta Silvestro ${ }^{\mathrm{a}}$ \\ ${ }^{a}$ Department of Neuroscience, University of Torino, Corso Raffaello 30, Torino 10125, Italy
}

\begin{abstract}
In biomedical studies as well as in clinical trials, it is often useful to have a reliable measure of the force exerted by the body (eg. clenching force at the teeth or pinch force at fingertips) or on the body by external stimuli (eg. taps to elicit reflexes or local pressure for nociceptive stimulation). Thin-film sensors such as FlexiForce ${ }^{\circledR}$ provide a very handy and versatile solution for these application, but can be easily damaged and offer poor accuracy and repeatability, being heavily affected by the surface material they get in contact with. The aim of the study is the realization of a 3D-printed cover that completely embeds the sensor, thus providing mechanical protection and increasing reliability of the measurement. The increasing availability of 3D printers and of printing materials for medical use allows the user to shape the cover according to specific needs, with short developing time and low cost.
\end{abstract}

Keywords: Thin Sensor, 3D FDM/FFF Print, Biomedical Tests.

\section{Introduction}

Thin-film force sensors are versatile and can potentially be employed in a wide range of applications. For example, due to their low thickness, they have been adopted for the measurement of force and pressure exerted on different body parts [1, 2, $3,4,5]$ as well as clenching force $[6,7,8,9]$. However the behavior of film sensors is strongly influenced by test conditions, e.g., area and nature of the contact surfaces, load direction, etc. [10,11]. The difficulty in maintaining the same working conditions during the calibration procedure and during the subsequent measurement sessions may impair accuracy and reliability of the measurements. Indeed, in a wide range of applications from orthodontics to physiotherapy, the sensors can come into contact with soft or hard parts of the human body (skin or teeth), with fluids (e.g. saliva), with concentrated loads (for example, teeth occlusion forces) and unknown tangential forces. On this basis, these sensors would benefit of a shield that protects them from structural damage while, at the same time, providing a stable interface, that reduces the effect of changes in stiffness and texture of contact materials. We here provide a possible solution, consisting of fully embedding the sensor in a 3D printed rigid cover, using a 3D FDM/FFF printing process. This approach is based on printing the cover around the film sensor, which, besides granting constant contacting interface and mechanical protection, also allows for designing complex cover shapes, which can fit different applications and purposes. We hypothesized that the 3D-printed cover would not alter the basic load-response curve of the sensor, but would reduce the dependency of the measurement on the contact surface, thus increasing its reliability. We also aimed at testing the performance of three different materials for the cover: PLA (Polylactic Acid), nGen Flex (ColorFabb, Polymer Amphora Flex FL6000) and ABS (Acrylonitrile Butadiene Styrene). PLA and ABS are the most used FDM printing materials. PLA is also a bioplastic, so its biocompatibility and its biodegradability make it very interesting for the biomedical sector. nGen Flex is a more flexible and softer material and it is appropriate for biomedical applications.

\section{Methods}

\subsection{Thin-film sensors}

The FlexiForce sensors (TekScan) have been employed in the present study. These are thin-film sensors that use a resistive based technology such that the application of a force to the active sensing area of the sensor results in a change in the resistance of the sensing element [12]. Every sensor has to be preconditioned before use and the construction of a calibration curve is recommended to account for the individual sensitivity of the transducers [12,13], provided that the same sensor configuration 
is used for calibration and for the actual measurements. The use of a puck is recommended from the manufacturer and in clinical tests its use is practically always necessary [14]. The sensor must therefore be incorporated into a shell with an upper and lower protection puck, 2 double-sided adhesive tape layers to avoid slipping between the sensor and the puck. The external shell should then be sufficiently hard to protect the sensor, while still allowing for correct sensor loading. Based on these recommendations the sensors have been embedded in a 3D-printed cover, as described below.

\section{2. $3 D$ printing}

The protecting cover is 3D-printed around the sensor, following the normal printing process steps. The cover is designed with the software Autodesk Fusion 360 with the aim of fixing the sensor in a stable position, while ensuring that the force lines of the applied load are conveyed through its sensing area. This latter issue is achieved by the circular cantilevered area (see inferior side of the cover in Fig.1(a)), matching the inner $80 \%$ of the sensing area $[12,14]$.
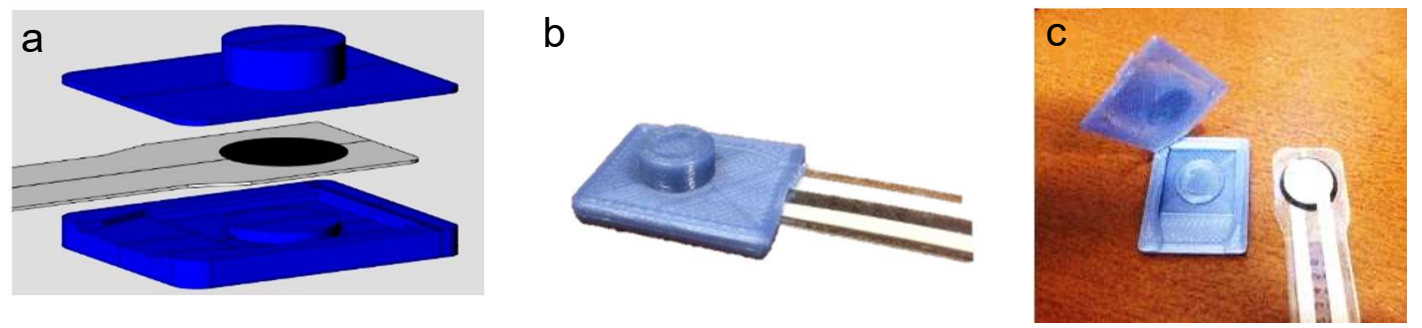

Fig.1. (a) 3D model of the cover realised with the software Autodesk Fusion 360. Note how the film sensor (in grey) is positioned over the bottom part of the cover, before the upper part is printed on top; (b) example of covered sensor; (c) upper and lower side of the cover have been separated with a cutter to show inner side. The material in this example is PLA.

The slicer software is IRA 3D (Cura). The 3D printer is IRA Poetry2 Dual Extrusion (2015). The printing temperature is set to $200^{\circ}$ for PLA, $250^{\circ}$ for $\mathrm{ABS}$, and $225^{\circ}$ for nGen Flex. Fortunately, they are materials with different printing temperatures, so the full range of normal printing temperatures is covered. Printing velocities are set in the range of $30-40 \mathrm{~mm} / \mathrm{s}$ and the thickness layer for all the printing processes is $0.2 \mathrm{~mm}$, which yields a satisfactory printing quality. Printing speed results from a trade-off between printing accuracy and the risk of overheating the sensor: it is preferable to have high speeds and low printing temperatures, to avoid damaging the sensor. The cooling action of the fans may help. The printing is automatically stopped at completion of the lower part of the cover and the FlexiForce sensor is then inserted and blocked in its position by means of double sided tape. Then, the double-sided tape, placed on the top face of the sensor, improves adhesion and the top of the cover is printed on the sensor. The upper layer of envelope is $0.6 \mathrm{~mm}$ (3 layers), which guarantees adequate mechanical protection to the sensor. It is possible to reduce the layers to 2, when the applied loads are small. Making less than 2 layers may not be sufficient to ensure adequate mechanical resistance of the cover. A cylindrical tip ( $2.4 \mathrm{~mm}$ in height) on the upper side further strengthens the structure and concentrates the load on the sensitive area of the FlexiForce. The total thickness of the printed cover is $5.4 \mathrm{~mm}$. The printing time is about 5 minutes.

\subsection{Experimental set-up}

Load response curves of naked and covered sensors were constructed by means of the set-up depicted in Fig. 2. The sensor was connected to a circuit for conditioning and amplification $[12,13]$, whose output was fed into a signal acquisition board (Micro1401, CED, UK) along with the calibrated output of a load cell (YZC-516 $50 \mathrm{Kg}$ ). Acquired signals were digitally transmitted and stored on a personal computer. A clamp was used to manually generate the load: within the clamp the sensor was positioned "in series" with the load cell, so that both simultaneously shared the same load when the clamp was tightened. A puck was positioned between the sensor and the load cell. Pucks of 3 materials with different stiffness (Table1) were used: Steel, PLA and Nitrile Butadiene Rubber (NBR). The contact surfaces are as smooth as possible, so as not to damage the naked sensor and not to worsen the detection of the applied load.

Table 1. Materials used for 3D printing or as puck

\begin{tabular}{llll}
\hline Material & & Used & Young Modulus (Mpa) \\
\hline PLA & Polylactic Acid & 3D Print Filament/ Puck & $3000-3500$ \\
nGen Flex & Polymer Amphora Flex FL6000 & 3D Print Filament & $80-100$ \\
ABS & Acrylonitrile Butadiene Styrene & 3D Print Filament & $2000-2500$ \\
Steel & Steel & Puck & 210000 \\
NBR & Nitrile Butadiene Rubber & Puck & 3.5 \\
\hline
\end{tabular}


Fig.2. Scheme of the experimental setup. LC: load cell, AMP: signal conditioning and amplification for both the sensor and the load cell.

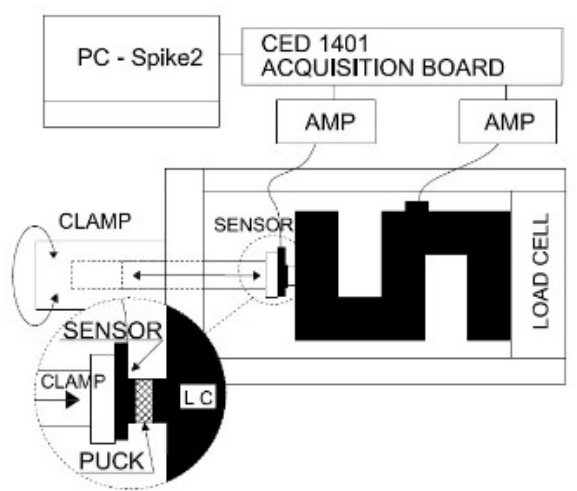

\subsection{Experimental Protocol}

Nine FlexiForce film sensors (model A201, $100 \mathrm{lbs}$, TekScan) were employed. The first three sensors (1-3) were covered with PLA, the second three (4-6) with nGenFlex and the last three (7-9) with ABS. Each sensor was tested in three subsequent conditions: i) without the cover (Naked), ii) after application of the cover (Cover I); after removal of the first cover and application of a new cover (Cover II). In each condition, the sensor was loaded through pucks of three different materials (Steel, PLA, NBR). In each of these 9 conditions the load response curve was recorded by manually slowly increasing the load from 0 to $40 \mathrm{~kg}$ (about $90 \%$ of the full scale) and then decreasing back to $0 \mathrm{~kg}$. Prior to each measurement session, the naked and covered sensors were preconditioned, according to the manufacturer's guidelines.

\subsection{Analysis and statistics}

The Hysteresis (\%) has been calculated as the maximum difference between the loading and unloading curve divided the maximum value of the output voltage (maximum load applied $=40 \mathrm{~kg}$ ). The Linearity has been evaluated with the coefficient of determination $\mathrm{r}^{2}$ for a trend line calculated with the minimum squares and intercept equal to 0 (maximum load applied $=$ $40 \mathrm{~kg}$ ). The Linear Mixed Model has been chosen as statistical method to compare the different working conditions (sensor output at $40 \mathrm{~kg}$ ), because the study presents both repeated measures both hierarchical structures of the data. Variability of the output due to different pucks was quantified by the coefficient of variation ( $\mathrm{CoV}=\mathrm{SD} / \mathrm{mean})$. The $\mathrm{CoV}$ was compared in the three different sensor conditions by means of the student-t test. The software statistical used is R 4.10.

\section{Results}

Representative examples of a load-response curve of a naked sensor tested with pucks of different materials are reported in Fig.4 (a). It can be observed that, although the curves are qualitatively similar, the magnitude of the response (i.e. the sensitivity of the sensor) is affected by the puck. In comparison, the covered sensor presents a remarkably similar response irrespective of the puck (Fig.4 (b)). Similar results were obtained for PLA and nGen Flex materials. On the contrary, no result is reported for $\mathrm{ABS}$ because the printing process damaged all 3 sensors, possibly due to the higher printing temperatures.
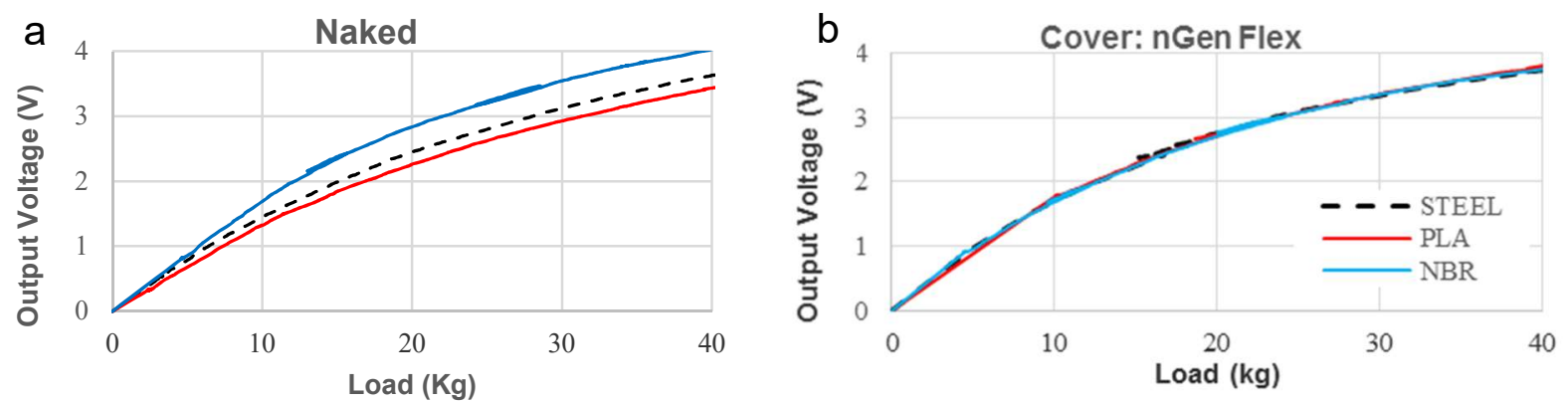

Fig.3 - (a) load-response curves for naked sensor tested with pucks of different materials. For the sake of clarity, only the loading part of the curve is shown; (b) load-response curves for covered sensor (nGen Flex) tested with pucks of different materials. For the sake of clarity only the loading part of the curve is shown 
The sensor output at $40 \mathrm{~kg}$ loading was used to compare the responses in the different sensor conditions (Naked, Cover I, Cover II) and with different contact materials (Steel, PLA, NBR). The results for the 3 sensors covered with PLA have shown that the sensor output did not depend on the sensor condition nor on the puck material (Fig.4 (a)). In fact, it can be observed that the presence of the cover does not alter the output of the sensor in a systematic way and the same applies to the puck material. However, it can be observed that the variability of the measurements due to different pucks is higher in the naked, compared to the covered conditions. The results are qualitatively similar for the 3 sensors covered with nGen-Flex, as shown in Fig.4 (b): again it can be observed that the puck largely affects the variability of the sensor output, but only in the naked condition. It can be observed that in some cases the cover increased the sensitivity of the sensor, with an increase in output voltage at a given load, in other cases the sensitivity was decreased. These differences may be attributed to small differences in the printing process.
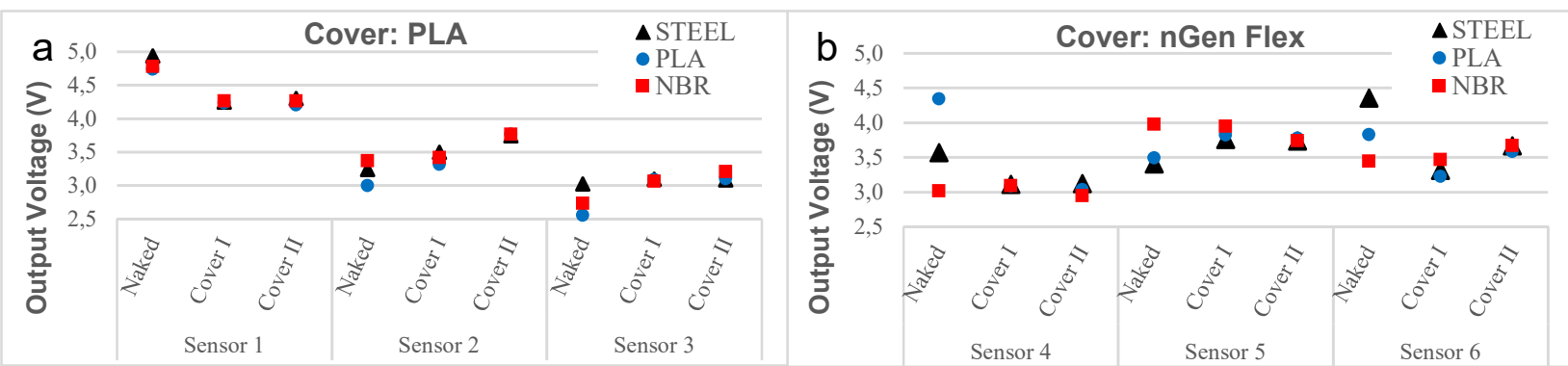

Fig. 4 - (a) response of the sensors to a 40-kg load when tested with pucks of different materials (Steel, PLA, NBR) in the different conditions (Naked, Cover I, Cover II) for cover made of PLA; (b) for cover made of nGen Flex. Note the spread of the responses in the naked condition.

The Linear Mixed Model has been used to break down the variance between-group, due to the specific sensitivity of each sensor, to the variance within-group due to the variation of the contact surface of puck (Steel, PLA, NBR), using the sensor condition as factor grouping (Naked, Cover I and Cover II). The assumption of normality of residuals was verified by the Shapiro-Wilk test. The heteroscedasticity of the variances between-group and within-group have been studied with a loglikelihood test (REML Restricted Maximum Likelyhood). The models (Model $\mathrm{Ho}_{\mathrm{He}}$ ) with homogeneity of between variance and heteroscedasticity for within variance are significantly better than models (Model $\mathrm{Ho}_{\mathrm{Ho}}$ ) with homogeneity of between and within variance (Naked-Cover I, Model $l_{\mathrm{Ho}-\mathrm{Ho}}$ to Model $_{\mathrm{Ho}-\mathrm{He}}$ : AIC 37 to $18, \sigma_{\mathrm{w}}{ }^{2} \mathrm{NAKED}=0,104-\sigma_{\mathrm{w}}{ }^{2} \mathrm{COVER} \mathrm{I}_{\mathrm{I}}=0,005, \mathrm{p}_{\mathrm{value}}<0.001$ - Naked-Cover II, Model $\mathrm{Ho}_{\mathrm{Ho}}$ to Model $\mathrm{Ho}_{\mathrm{He}}$ : AIC 37 to $\left.12, \sigma_{\mathrm{w}}{ }^{2} \mathrm{NAKED}=0,104-\sigma_{\mathrm{w}}{ }^{2} \mathrm{COVVR}_{\mathrm{II}}=0,003, \mathrm{p}_{\mathrm{value}}<0.001\right)$. The models $\left(\right.$ Model $\left._{\mathrm{He}-\mathrm{He}}\right)$ with heterogeneity of the between and within variance did not lead to significant improvements: the between variance is almost the same before and after 3D encapsulation, as the printing process can lead to improvements or worsening always however referred to the specific sensitivity of the sensor. The printing process does not alter the between variance and improves the within variance. The larger variability produced by the different pucks in the naked, compared to the covered conditions is quantified by the coefficient of variation CoV and shown in Fig. 5.

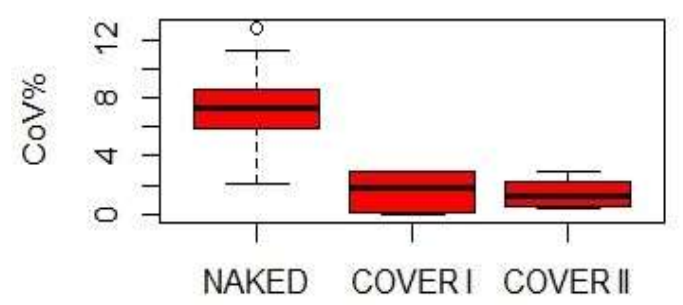

Fig.5: Coefficient on Variation ( $\mathrm{CoV}$ ) of the responses to 40-kg loading with pucks of different material in the different sessions. Error bars represent $95 \%$ confidence intervals.

In the naked condition the $\mathrm{CoV}$ is significantly higher than in the covered conditions (mean $\mathrm{CoV}_{\mathrm{NAKED}}=8,3 \%-$ mean $\mathrm{CoV}_{\text {COVER I }}=1,84 \%$ : $\mathrm{p}_{\text {value }}=0,007$; mean $\mathrm{CoV}_{\text {NAKED }}=8,3 \%$ - mean $\operatorname{CoV}_{\text {COVER II }}=1,10 \%$ : $\mathrm{p}_{\text {value }}=0,003$;). The hysteresis between loading and unloading curve was not prominent in naked sensors (4.8\% average over sensors 1-6) and was not affected by the cover (Cover I: $4.8 \%$, Cover II: $4,5 \%$ ). Also the linearity for the covered sensors $\left(r^{2}=0.90\right.$ average for Cover I, $r^{2}=0.92$ average for Cover II) is similar to the naked sensors $\left(r^{2}=0.89\right.$ average over sensors $\left.1-6\right)$.

\section{Discussion}

The study demonstrated the possibility of completely embedding a thin-film force sensor within a rigid 3D-printed cover, through a self-made 3D printing. The presence of a cover did not produce significant changes in the load-response curve of the sensor, in terms of sensitivity, linearity and hysteresis, but consistently reduced the dependence of the sensitivity on contact materials, thus greatly reducing the output variability in repeated measurements. While these sensors offer acceptable repeatability when tested always in the same condition [14], changes in the contact surface in terms of texture and compliance may profoundly affect the measurement $[10,11]$. Independence of the force measurement on the contact surface is a crucial need in biomedical applications, in which calibration of the sensor "in the same condition" in which it is experimentally 
employed (as recommended by the manufacturer) is not always possible [2, 3, 4, 5, 15]. Consequently, this aspect is often neglected and data collected may be highly imprecise. In this respect, 3D-printed covers may provide an easy solution, in fact the $\mathrm{CoV}$ was reduced to less than $2 \%$. Among the many biocompatible materials on the market, such as PLA and nGenFlex used in the study, you must choose those with lower printing temperatures, since too high temperatures may damage the sensor, as was shown to happen with ABS. Particular attention must be paid to find the optimal printing temperature to avoid bad adhesion phenomena between layers and to the shape of the cover to avoid the presence of tangential forces, not detectable by the sensor, and to give the right stiffness overall, to maintain the flatness of the loading area. Only repeated printing tests can lead to the correct choices. Fortunately, the mechanical characteristics of the printing materials and the modest forces applied or applicable in the biomedical field (in any case not exceeding 500N for the sensors used) should allow designing the cover in safety. In the case of concentrated forces, such as for the forces transmitted by the teeth, it is important to interpose casts (for example with material for dental use) on the cover properly designed, to stabilize and distribute the forces. High performance printers makes it possible also to create very complex models, that adapt to precise anatomical parts thus allowing for easy positioning and use. The sensor cover also protects it from accidental contact with fluids, such as sweat and saliva, as the sensor risks being irreparably damaged. Unfortunately, small imperfections in the printing phase (with our old printer) make them not adaptable to the immersion of the encapsulated sensor. We have conducted various tests by immersing the sensor as completely encapsulated along its entire length in isopropanol $75 \%$ several times for 5 minutes in order to sterilize it [16]. Unfortunately, after a few dives the sensors were damaged. Therefore the only way to sterilize the cover sensor, not even being able to use steam or high temperatures, is to use UV-C rays $30 \mathrm{~W}$, wavelength less than $280 \mathrm{~nm}$, $15 \mathrm{~min}$, as suggested by Prusa [16], after having thoroughly cleaned it of biological substances (e.g. saliva) with an absorbent cloth and then with cotton soaked in disinfectant (e.g. isopropanol 75\%). The sterilization process must be performed once for each sensor face.

\section{REFERENCES}

[1] A. Botter, T. M. Vieira, T. Geri, and S. Roatta (2020), "The peripheral origin of tap-induced muscle contraction revealed by multi-electrode surface electromyography in human vastus medialis," Sci. Rep., vol. 10, no. 1, pp. 1-11, doi: 10.1038/s41598-020-59122-z.

[2] Berwin J T, Burton T M W, Taylor J, Mcgregor A H, Roche A (2015), "Plantar loading forces while walking in a below-knee cast with an attached loadbearing frame," Foot Ankle Int., vol. 36, no. 6, pp. 722-729, doi: 10.1177/1071100715572258.

[3] Liu R, Kwok Y L, Li Y, Lao T T H, Zhang X, Dai X Q (2005), “Objective evaluation of skin pressure distribution of graduated elastic compression stockings," Dermatologic Surg., vol. 31, no. 6, pp. 615-624, doi: 10.1097/00042728-200506000-00001.

[4] Pais Clemente M, Mendes J, Cerqueira J, Moreira A, Vasconcelos M, Ferreira A P, Amarante J M (2019), "Integrating piezoresistive sensors on the embouchure analysis of the lower lip in single reed instrumentalists: implementation of the lip pressure appliance (LPA)," Clin. Exp. Dent. Res., vol. 5, no. 5, pp. 491-496, doi: 10.1002/cre2.214.

[5] Parthiban S, Hotaling J M, Kathrins M, Baftiri A P, Freels S, Niederberger C S (2015), "A novel method to determine perineal artery occlusion among male bicyclists," PeerJ, vol. 2015 , no. 12 , pp. 1-11, doi: 10.7717/peerj.1477.

[6] Testa M, Geri T, Signori A, Roatta S (2015), "Visual Feedback of Bilateral Bite Force to Assess Motor Control of the Mandible in Isometric Condition," Motor Control, vol. 19, no. 4, pp. 312-24, doi: 10.1123/mc.2014-0011.

[7] Testa M, Rolando M, Roatta S (2011), "Control of jaw-clenching forces in dentate subjects," J Orofac Pain, vol. 15, no. 3, pp. 250-60, PubMed PMID: 21837292.

[8] Flanagan D, Ilies H, O'Brien B, McManus A, Larrow B (2012), “Jaw bite force measurement device,” J Oral Implantol, vol. 38, no. 4, pp. 361-4, doi:10.1563/AAID-JOI-D$10-00101$.

[9] H. W. Weisskircher (2013), "Maximum bilateral masticatory forces in patients with and without pain," Zeitschrift für Kraniomandibuläre Funktion, vol. 5, no. 1, pp. 19-39.

[10] Testa M, Di Marco A, Pertusio R, Van Roy P, Cattrysse E, Roatta S (2016). "A validation study of a new instrument for low cost bite force measurement," Journal of Electromyography and Kinesiology, vol. 30, pp: 243-248, doi: 10.1016/j.jelekin.2016.08.005.

[11] Likitlersuang J, Leineweber M J, Andrysek J (2017), "Evaluating and improving the performance of thin film force sensors within body and device interfaces," Med. Eng. Phys., vol. 48, pp. 206-211, doi: 10.1016/j.medengphy. 2017.06.017.

[12] Tekscan (2016), "FlexiForce $\circledR$ Sensors User Manual," Tekscan Inc, pp. 1-15, [Online].

[13] Tekscan, "Best Practices in Electrical Integration of the Flexi Force TM Sensor," Tekscan, [Online].

[14] Tekscan, "Best Practices in Mechanical Integration of the Flexi Force TM Sensor," Tekscan, [Online].

[15]Valentim A F, Furlan R M M M, Perilo T V de C, Berbert M C B, Motta A R, de Las Casas E B (2014), "Evaluation of the force applied by the tongue and lip on the maxillary central incisor tooth," CoDAS 2014, vol. 26, no. 3, pp. 235-240, doi: 10.1590/2317-1782/201420130077.

[16] Prusa Face Shield disinfection, "https://help.prusa3d.com/en/article/prusa-face-shield-disinfection_125457 